\title{
Maximizing the electron field emission performance of carbon nanotube arrays
}

\author{
R. C. Smith ${ }^{\text {a) }}$ and S. R. P. Silva \\ Nano-Electronics Centre, Advanced Technology Institute, Faculty of Engineering and Physical Sciences, \\ University of Surrey, Guildford, Surrey GU2 7XH, United Kingdom
}

(Received 5 January 2009; accepted 11 February 2009; published online 1 April 2009)

\begin{abstract}
The field emission screening effect is one of great importance when aiming to design efficient and powerful cathodes. It has long been assumed that the degrading effect is at a minimum when neighboring emitters are at least twice their height from each other. In this work, we show that the screening effect is underestimated and diminishes at far greater separations of five times the height of the emitter. We further observe that to achieve maximum emission efficiency in an array, one requires a trade off between screening and emitter number, resulting in a separation of three times their height. (C) 2009 American Institute of Physics. [DOI: 10.1063/1.3097239]
\end{abstract}

The use of carbon nanotubes (CNTs) as electron sources for field emission (FE) applications ${ }^{1,2}$ has generated a wide interest since their identification in 1991. The ability to grow ordered arrays of aligned CNT on a range of substrates has made it possible for designers to specify height, radius, separation, etc., and for these requirements to be accurately fabricated. However, the exact process of electron emission from CNTs is still not fully understood. Numerous papers investigating the field enhancement factor of an individual CNT have attempted to further the understanding of the emission process through experimental and computational investigations into geometric enhancement factor. ${ }^{4-9}$ It has long been assumed that the effect of field screening is negated when adjacent emitters are at a separation of twice their height. ${ }^{6}$ In this letter, we investigate the effect of field screening on arrays of CNT by means of three-dimensional (3D) simulations and show the impact on device performance.

CNTs were simulated in 3D in SIMION 8.0 software. $^{10}$ The software solves the Laplace equation using finite difference with optimized linear time solving to allow extraction of potential and electric field at any point around the structure. An initial calibration simulation was that of a hemisphere on a grounded plane, beneath an anode at a positive potential. Numerically, the solution to the Laplacian equation in $3 \mathrm{D}$ for such a structure gives a local field above the hemisphere of three times that of the applied field placed infinitely far away. It was found that as long as the anode electrode was maintained sufficiently afar as not to incur anode proximity effects, ${ }^{8,9}$ the enhancement factor simulated was 3.01 .

Figure 1(a) shows a schematic of the simulated array of CNT. Vertically aligned CNTs of uniform height $h$ and radius $r$ are placed on a grounded cathode that is separated from the positive anode by distance $D$. To simulate a realistic CNT array the impact of the size of the array on screening was examined. We simulated five different conditions. Firstly, a single CNT of height $3 \mu \mathrm{m}$ and radius $50 \mathrm{~nm}$ was placed at the center of the simulation work space. The simulation package has a maximum of $200 \times 10^{6}$ calculation points. This places an upper limit on the array dimensions for simu-

${ }^{a)}$ Electronic mail: r.c.smith@surrey.ac.uk. lation to ensure that the anode was sufficiently far away from the CNT and that the CNT occupies sufficient points to ensure the tip is suitably rounded. We also ensured our array structures were positioned adequately far from the edges of the simulation work area to avoid unwanted boundary effects. The other four scenarios were the single CNT with two adjacent neighbors, then four, six, and eight neighbors. All intertube separations were kept constant at $3 \mu \mathrm{m}$. It was ob-

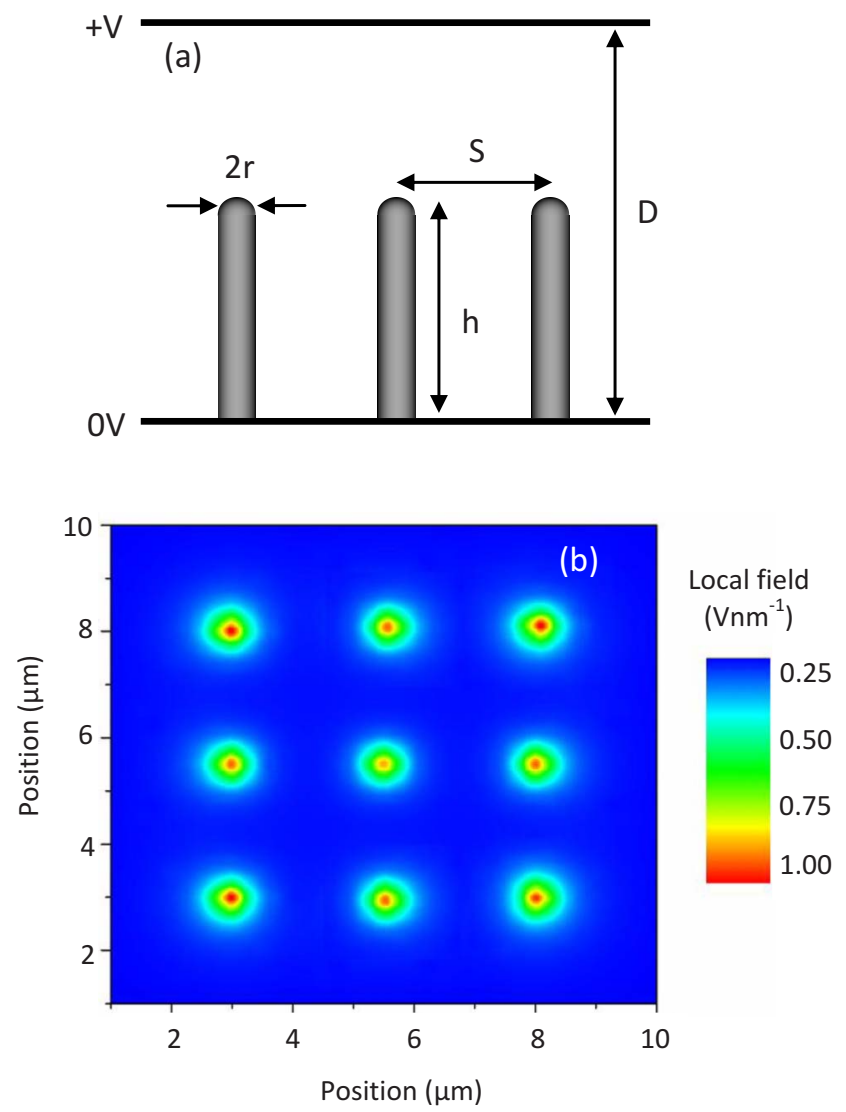

FIG. 1. (Color online) (a) Schematic of the simulation setup of a single CNT of height $h$ and radius $r$, placed on a grounded cathode, and beneath an anode at a distance $D$ away. (b) Contour plot of local electric field for an array of nine equally spaced CNT of height $3 \mu \mathrm{m}$ and radius $50 \mathrm{~nm}$. The spacing between them is $3 \mu \mathrm{m}$. It can be seen in this top down view that the CNT at the corners have the highest local field, followed by the CNT in the outer centers, with the middle CNT having the lowest local field. 


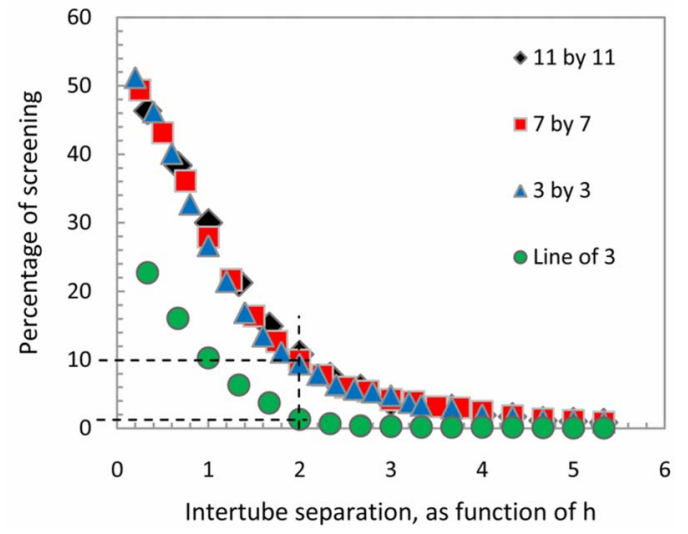

FIG. 2. (Color online) Percentage of screening calculated by the difference in local electric field between an isolated CNT, to the middle CNT of an array of 3 by $3(\boldsymbol{\Lambda}), 7$ by 7 ( $)$ and 11 by 11 CNT ( ). At $S=2 h$, the middle CNT is screened by approximately $11 \%$ for all three cases. For comparison, three CNT each spaced by $3 \mu \mathrm{m}$ was simulated in $2 \mathrm{D}(\bullet)$. Here it is seen that at $S=2 h$ the screening factor is less than $2 \%$.

served that the ratio of the local fields of the central CNT in the array to the isolated CNT varied only by $1.5 \%$ for all simulations. This implied that from here on we can simulate an array of nine CNTs and still be confident about the accuracy of our results.

The as simulated array of nine CNTs is shown as a top down view to Fig. 1(b) where the CNTs are separated by a distance $S$. An anode to cathode separation of $80 \mu \mathrm{m}$ was used, and a positive potential of $100 \mathrm{~V}$ applied to the anode. The local field was carefully extracted at a distance immediately above the middle CNT. It can be seen that the middle CNT has a lower local field than that of its neighbors which is approximately $85 \%$ of the field experienced by the emitters on the corners of the array. It was also noted on that the local field at the tip of the corner CNTs were still lower by $8.6 \%$ than that of the isolated control simulation. ${ }^{11}$

It is known that two emitters in close proximity will reduce the local field due to screening. It has long been assumed the optimum intertube separation is twice the height of the CNT, or, $S=2 h .^{6}$ This observation has featured in many publications interested in electron field emission from $\mathrm{CNT},{ }^{12-14}$ and in some cases given reference as a to a low understanding of field screening. ${ }^{15}$ Our array of CNTs seen in Fig. 1(b) was simulated for intertube separations $S$ ranging from 1 to $16 \mu \mathrm{m}$, which corresponds to $S=0.3 \mathrm{~h}$ to $5.3 \mathrm{~h}$. A percentage of screening was calculated by the difference in the local field for the middle CNT to that of an identical but isolated CNT. Figure 2 shows the percentage of screening for CNTs of radius $50 \mathrm{~nm}$, height $3 \mu \mathrm{m}$. It is observed that the amount of screening decreases as $S$ increases However, the amount of screening at $S=2 h$ (shown by the dotted lines) indicate that there is still a 10\% drop in local field. It is important to state that while in our simulations, only the central CNT is screened the most, in a much larger array over the ranges of $\mathrm{mm}^{2}$ this $10 \%$ screening would be applicable to all CNT except those at the outer edge, as demonstrated for an array of 11 by 11 CNT shown in Fig. 3. It can be seen that the majority of emitters within the center of the array exhibit similar fields. The 10\% drop in local field means that at $S=2 h$, the vast majority of the array is performing at $90 \%$ of its optimum value. To achieve a fully unscreened array of CNT, one needs to have an intertube separation in excess of five times the height of the CNT. This is particularly important when investigating individual CNT within an array. To understand where the underestimate of screening arises, we simulated a "line" of three CNTs, which approximates a two-dimensional (2D) scenario to mimick the work of Nilsson et al., ${ }^{12}$ while keeping the same dimensions as before. This is shown in Fig. 2 as closed circles. In this case, at $S=2 h$, the percentage of screening was found to be $<2 \%$, which informs on the origin of the (now underestimated) assumption that screening is at a minimum at $S=2 h$. However, we believe that our study of 3D arrays of CNT to be more applicable to experimentally grown ordered arrays of emitters than the results obtained from 2D simulations.

As discussed previously, in a large array of emitters, the screening effect is less at the edge of the array, where the emission current will be higher than those within the center. Figure 3 illustrates this effect by showing the local field above and surrounding a larger emission area comprising of 121 emitters. It is desirable in many field emission applications to have uniform emission over the entire active area. By using the effect of screening seen thus far, we can adjust the position of emitters at the outermost levels to increase the uniformity of the array. To compensate for this, we move the second layer of emitters inwards by approximately $10 \%$ and the outside layer emitters in by approximately 20\% (not shown). The knock-on effect of this is a leveling of the local electric field. While the third layer of emitters was not moved, it was observed that there was a slight drop in field due to the movement of the first and second layers. It was also noticed that the corner emitters still had a slightly higher local field. To reduce this, one needs to move the corners in further to maintain a constant screening between every emitter in the array.

It is important to consider the effect of screening from a practical point of view. In order to quantify the impact of our findings, we take values of CNT dimensions and FE performance from previous work ${ }^{8}$ and model an array of $5 \mathrm{~mm}^{2}$, at varying intertube separations.

Taking the standard Fowler-Nordheim type relationship, but using local field, $E_{L}$ in place of $\beta E$ we get
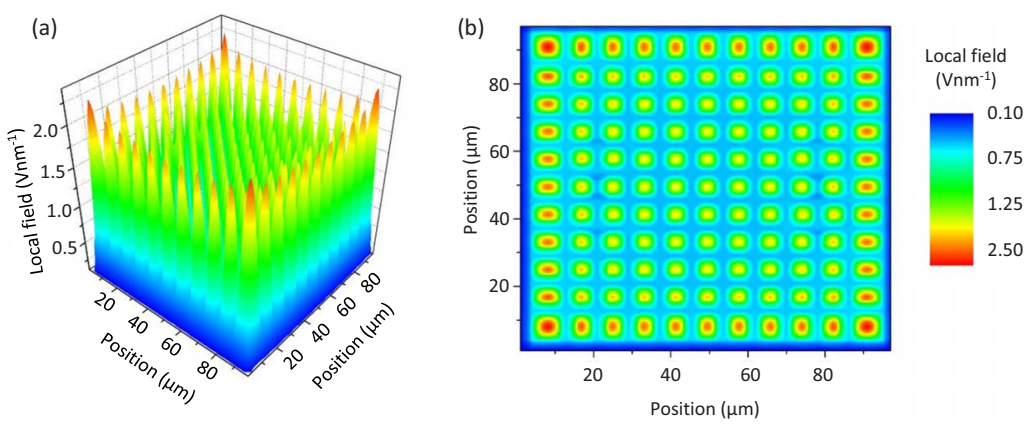

FIG. 3. (Color online) (a) Contour plot of local electric field over an array of 11 by 11 CNT with a spacing of $S=h$. The central area of the array two rows in, all exhibits a similar screening factor. (b) Top down view of the same scenario. The outermost two layers of CNT have a local field that is $35 \%$ higher than the majority of the array. 

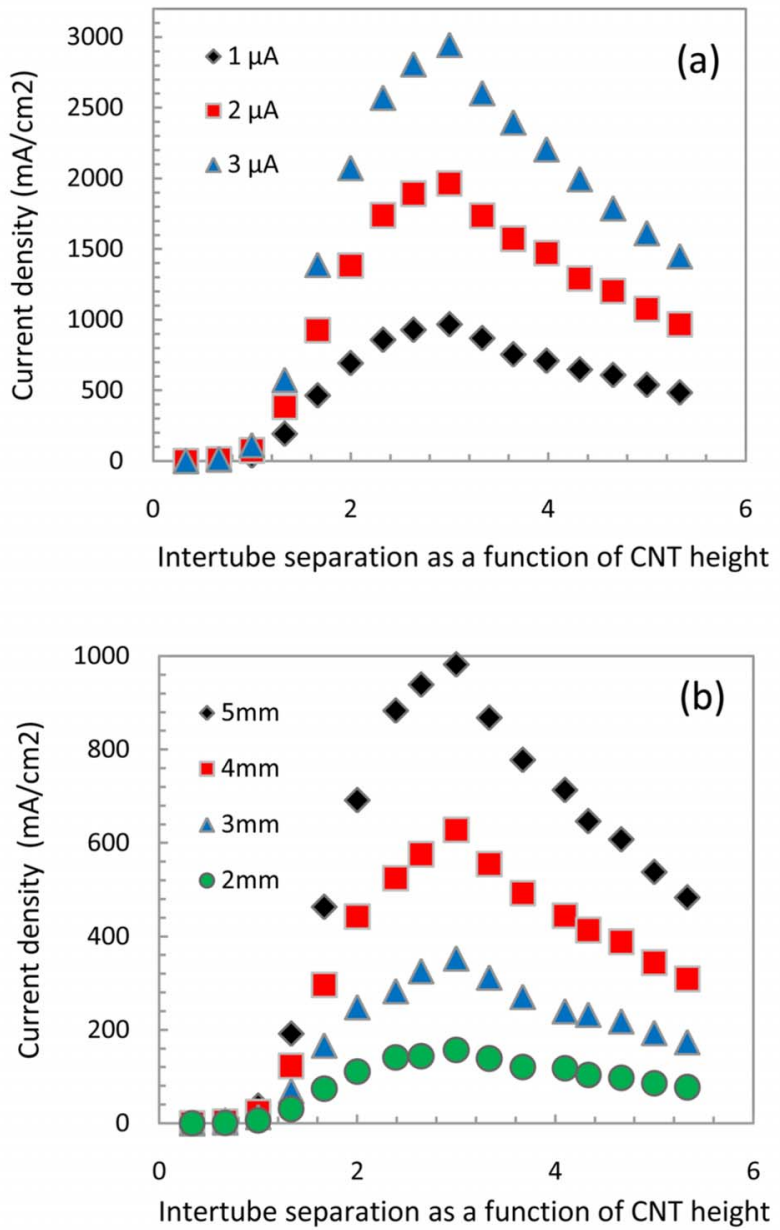

FIG. 4. (Color online) (a) Current density calculated for an array size of $5 \mathrm{~mm}^{2}$ and at varying CNT separations. A maximum current density is observed at $S=3 h$, even though the local field at each CNT is reduced by $3.8 \%$ due to screening. Above values of $S$ of 3.5, a linear decrease in current density is observed. The isolated CNT used in Eq. (3) has an emission current of $1 \mu \mathrm{A}(\diamond), 2 \mu \mathrm{A}(\boldsymbol{\square})$, and $3 \mu \mathrm{A}(\mathbf{\Delta})$. (b) Similar trends are seen for varying emission area of $5 \mathrm{~mm}^{2}(\diamond), 4 \mathrm{~mm}^{2}(\boldsymbol{\square}), 3 \mathrm{~mm}^{2}(\boldsymbol{\Lambda})$, and $2 \mathrm{~mm}^{2}(\bullet)$

$$
I_{0}=\frac{a A E_{L}^{2}}{\phi} \exp \left(\frac{-b \phi^{3 / 2}}{E_{L}}\right) .
$$

Comparing the ratio of local fields for an isolated CNT $\left(E_{L 1}\right)$ and of a CNT in an array $\left(E_{L 2}\right)$,

$$
E_{L 1}=S E_{L 2},
$$

where $S$, the screening factor, is between 0 and 1 . The ratio of emission current for isolated CNT $\left(I_{\text {isolated }}\right)$ and the screened CNT within the array $\left(I_{\text {screened }}\right)$ is

$$
\begin{aligned}
\frac{I_{\text {screened }}}{I_{\text {isolated }}} & =S^{2} \exp \left[\left(\frac{-b \phi^{3 / 2}}{E_{L \text { isolated }}}\right)+\left(\frac{-b \phi^{3 / 2}}{E_{L \text { screened }}}\right)\right], \\
& =S^{2}\left[\exp \left(\frac{-b \phi^{3 / 2}}{E_{L \text { isolated }}}\right)\right]^{[(1 / S)-1]},
\end{aligned}
$$

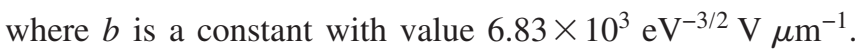
$\Phi$ is the work function with value $4.5 \mathrm{eV}$, and $E_{L \text { isolated }}$ is the local field, in $\mathrm{V} \mu \mathrm{m}^{-1}$ at the apex of the unscreened CNT, calculated from experimental results. ${ }^{8}$ Taking the two cases discussed so far, $S=2 h$ and $S=5 h$, we calculated an ordered array of CNT to be screened by $10 \%$ and $2 \%$, respectively
(Fig. 3), and assuming each (unscreened) CNT is capable of emitting a stable $1 \mu \mathrm{A}$, we see the screened emission $I_{1}$ has decreased to $177 \mathrm{nA}$ for $S=2 h$, and $861 \mathrm{nA}$ for $S=5 h$. To explore this effect more, we calculated the emission current density for arrays of ordered CNT that occupy an area of $5 \mathrm{~mm}^{2}$. Plotted in Fig. 4(a), we see a sharp increase in current density as the initially tightly packed CNT become more separated. A maximum current density can be observed when the intertube separation approaches three times the CNT height. Beyond this value, the current density decreases. The linear decrease beyond $S=3 h$ would be expected, as at these CNT separations each emitter is unscreened, i.e., half the CNT equals half the current density. Increasing the unscreened emission current to 2 and $3 \mu \mathrm{A}$, we see similar trends. This phenomenon is also present if we change the emission area. Decreasing from 5 to $2 \mathrm{~mm}^{2}$ [Fig. 4(b)] we see similar trends. We show for an ordered array of CNT the screening effect diminishes when the intertube separation exceeds $5 h$, but practically, the most efficient arrangement of emitters, for maximum current density per unit area, would actually be $3 h$, giving rise to a $4 \%$ screening of the local field.

In conclusion, we have simulated arrays of CNT emitters in $3 \mathrm{D}$ and investigated the effect of field screening in terms of intertube separation. We have found that the previous optimum separation of $2 h$ to be an under estimate of screening in $3 \mathrm{D}$, and to achieve a fully unscreened array, the optimum separation needs to be in excess of five times the height of the CNT. Conversely, we have shown that when considering arrays of emitters for practical applications, there is a compromise to ensure maximum efficiency in terms of current density of a separation of three times the CNT height. We also demonstrate that a fuller appreciation of the screening effect lead to more efficient arrays but also more uniform emitter areas.

The authors would like to thank the EPSRC Portfolio Partnership program.

${ }^{1}$ V. Semet, V. T. Binh, P. Vincent, D. Guillot, K. B. K. Teo, M. Chhowalla, G. A. J. Amaratunga, W. I. Milne, P. Legagneux, and D. Pribat, Appl. Phys. Lett. 81, 343 (2002).

${ }^{2}$ E. Minoux, O. Groening, K. B. K. Teo, S. H. Dalal, L. Gangloff, J. -P. Schnell, L. Hudanski, I. Y. Y. Bu, P. Vincent, P. Legagneux, G. A. J. Amaratunga, and W. I. Milne, Nano Lett. 5, 2135 (2005).

${ }^{3}$ S. Iijima, Nature (London) 56, 354 (1991).

${ }^{4}$ W. A. de Heer, A. Chatelain, and D. Ugarte, Science 270, 1179 (1995).

${ }^{5}$ Y. Saito, K. Hamaguchi, T. Nishino, K. Hata, and K. Tohji, Jpn. J. Appl. Phys., Part 2 36, L1340 (1997).

${ }^{6}$ J. M. Bonard, J. P. Salvetat, T. Stockli, L. Forra, and A. Chatelain, Appl. Phys. A: Mater. Sci. Process. 69, 245 (1999).

${ }^{7}$ C. J. Edgcombe and U. Valdre, J. Microsc. 203, 188 (2001).

${ }^{8}$ R. C. Smith, D. C. Cox, and S. R. P. Silva, Appl. Phys. Lett. 87, 103112 (2005).

${ }^{9}$ R. C. Smith, J. D. Carey, R. D. Forrest, and S. R. P. Silva, J. Vac. Sci. Technol. B 23, 632 (2005).

${ }^{10}$ Simion $^{\mathrm{TM}}$, Idaho National Lab (INL) and Scientific Instrument Services Inc.

${ }^{11}$ R. C. Smith, R. D. Forrest, J. D. Carey, W. K. Hsu, and S. R. P. Silva, Appl. Phys. Lett. 87, 013111 (2005).

${ }^{12}$ L. Nilsson, O. Groening, C. Emmenegger, O. Kuettel, E. Schaller, L. Schlapbach, H. Kind, J. -M. Bonard, and K. Kern, Appl. Phys. Lett. 76, 2071 (2000)

${ }^{13}$ J. S. Suh, K. S. Jeong, J. S. Lee, and I. Han, Appl. Phys. Lett. 80, 2392 (2002)

${ }^{14}$ M. Mauger, V. T. Binh, A. Levesque, and D. Guillot, Appl. Phys. Lett. 85, 305 (2004).

${ }^{15}$ J. M. Bonard, M. Croci, C. Klinke, R. Kurt, O. Noury, and N. Weiss, Carbon 40, 1715 (2002). 